\title{
GLOBAL PRESERVATION OF NODAL STRUCTURE IN COUPLED SYSTEMS OF NONLINEAR STURM-LIOUVILLE BOUNDARY VALUE PROBLEMS
}

\author{
ROBERT STEPHEN CANTRELL
}

(Communicated by Kenneth R. Meyer)

ABSTRACT. In this paper, we examine the solution set to the coupled system

(*)

$$
\left\{\begin{array}{l}
-\left(p_{1}(x) u^{\prime}(x)\right)^{\prime}+q_{1}(x) u(x)=\lambda u(x)+u(x) \cdot f(u(x), v(x)) \\
-\left(p_{2}(x) v^{\prime}(x)\right)^{\prime}+q_{2}(x) v(x)=\mu v(x)+v(x) \cdot g(u(x), v(x)),
\end{array}\right.
$$

where $\lambda, \mu \in R, x \in[a, b]$, and the system $(*)$ is subject to zero Dirichlet boundary data on $u$ and $v$. We determine conditions on $f$ and $g$ which permit us to assert the existence of continua of solutions to $(*)$ characterized by $u$ having $n-1$ simple zeros in $(a, b), v$ having $m-1$ simple zeros in $(a, b)$, where $n$ and $m$ are positive but not necessarily equal integers. Moreover, we also determine conditions under which these continua link solutions to $(*)$ of the form $(\lambda, \mu, u, 0)$ with $u$ having $n-1$ simple zeros in $(a, b)$ to solutions of $(*)$ of the form $(\lambda, \mu, 0, v)$ with $v$ having $m-1$ simple zeros in $(a, b)$.

\section{INTRODUCTION}

In a previous paper [3], we established a theorem which gives sufficient conditions for higher global bifurcation in certain several-parameter systems of nonlinear eigenvalue problems. The systems we considered had the particular form

$$
A_{i} u_{i}=\lambda_{i} u_{i}+u_{i} \cdot f_{i}\left(u_{1}, \ldots, u_{n}\right),
$$

$i=1,2, \ldots, n$. For each $i \in\{1,2, \ldots, n\}, u_{i} \in E$, a commutative real Banach algebra, while for some subspace $D$ of $E, A_{i}: D \rightarrow E$ is an invertible linear operator with compact inverse $A_{i}^{-1}$.

In this paper, we examine a special case of (1.1). Namely we consider the system

$$
\begin{aligned}
& -\left(p_{1} u^{\prime}\right)^{\prime}+q_{1} u=\lambda u+u \cdot f(u, v), \\
& -\left(p_{2} v^{\prime}\right)^{\prime}+q_{2} v=\mu v+v \cdot g(u, v)
\end{aligned}
$$

Received by the editors September 13, 1988.

1980 Mathematics Subject Classification (1985 Revision). Primary 34B15; Secondary 34B25, $58 \mathrm{E} 07$.

This work was partially supported by the National Science Foundation under grant \#DMS8802346. 
on the bounded interval $(a, b)$ subject to the boundary conditions $u(a)=0=$ $u(b), v(a)=0=v(b)$. (More general homogeneous boundary conditions will not affect the validity of most of our results. However, we do need the same boundary conditions on $u$ and on $v$ in order for (1.2) to be an example of (1.1).)

We demonstrate that under reasonably general conditions on $f$ and $g$ for any pair of nonnegative integers $(n, m)$, there exist continua of componentwise nontrivial solutions $(u, v)$ to (1.2) where $u$ has $n$ simple zeros in $(a, b)$ and $v$ has $m$ simple zeros in $(a, b)$. These continua arise as secondary bifurcations as the parameters $\lambda$ and $\mu$ are varied from solutions to (1.2) of the form $(\tilde{u}, 0)$ or $(0, \tilde{v})$ where $\tilde{u}$ is a solution to

$$
\begin{aligned}
-\left(p_{1} u^{\prime}\right)^{\prime}+q_{1} u & =\lambda u+u \cdot f(u, 0), \\
u(a) & =0=u(b),
\end{aligned}
$$

with $n$ simple zeros in $(a, b)$ and $\tilde{v}$ is a solution to

$$
\begin{aligned}
-\left(p_{2} v^{\prime}\right)^{\prime}+q_{2} v & =\mu v+v \cdot g(0, v), \\
v(a) & =0=v(b),
\end{aligned}
$$

with $m$ simple zeros in $(a, b)$. Moreover, under additional hypotheses we show that the $(\tilde{u}, 0)$ and $(0, \tilde{v})$ solutions to $(1.2)$ are linked together by the continua whose existence we establish.

We should briefly note that this phenomenon has been observed in ([2], [5]) in the componentwise positive solutions to the system

$$
\begin{aligned}
-\Delta u & =u[a-u-c v] & & \\
-\Delta v & =v[d-e u-v] & & \text { in } \Omega, \\
u & \equiv 0 \equiv v & & \text { on } \partial \Omega,
\end{aligned}
$$

where $\Omega \subseteq \mathbf{R}^{N}$ is a smooth bounded domain. Indeed, it was work on (1.5) which initially motivated [3]. The system (1.5) is a steady-state Lotka-Volterra competition model with diffusion. Consequently, the bifurcation phenomenon may be described physically as follows. If $c$ and $e$ are fixed positive numbers, componentwise positive solutions to (1.2) (the coexistent steady-states $(u, v)$ ) arise as secondary bifurcations from solutions with one positive component and one trivial component (the so-called extinction states $(u, 0)$ and $(0, v)$ ) by varying the parameters $a$ and $d$. Moreover, the coexistence states form a sheet linking the extinction states of the form $(u, 0)$ to the ones of the form $(0, v)$.

We should also note that the results of this paper are in sharp contrast to the results of [4]. In [4], a pair of nonlinear Sturm-Liouville boundary value problems which depend on two parameters $\lambda$ and $\mu$ and which are coupled in the linearization about $(u, v)=(0,0)$ is demonstrated to possess continua of componentwise nontrivial solutions where the components change nodal structure. The difference reflects the effects of the stronger coupling in the systems 
considered in [4]. It also serves as a reminder of the endless fascination that coupled systems of nonlinear boundary problems of ordinary and partial differential equations provide.

Finally, we structure the remainder of this paper as follows. In Section 2, we determine conditions on $f$ and $g$ which guarantee the existence of continua of solutions to (1.2) whose first component has $n-1$ simple zeros in $(a, b)$ and whose second component has $m-1$ simple zeros in $(a, b)$ for any choice of $(n, m) \in\left(\mathbf{Z}^{+}\right)^{2}$. Then in Section 3, we give additional conditions on $f$ and $g$ to have these continua link solutions to (1.2) where the first component has $n-1$ simple zeros in $(a, b)$ and the second component is identically zero to solutions of (1.2) with the first component identically zero and the second having $m-1$ simple zeros in $(a, b)$. We conclude in Section 4 with a specific example which illustrates the results of Sections 2 and 3.

\section{BACKGROUND AND MAIN RESULT}

Let us recall the basic facts regarding nonlinear Sturm-Liouville boundary value problems which will be of importance to our analysis. (For details, see, for example, [6].) Let $[a, b]$ be a closed bounded interval and let $p, q:[a, b] \rightarrow \mathbf{R}$ be functions with the properties that $p \in C^{1}[a, b]$ and $p(x)>0$ for $x \in[a, b]$ and $q \in C[a, b]$. Let $h: \mathbf{R} \rightarrow \mathbf{R}$ be a continuous function with the properties that $h(0)=0$ and that $h(S)$ is a bounded set whenever $S \subseteq \mathbf{R}$ is a bounded set. Then the nonlinear Sturm-Liouville boundary value problem

$$
\begin{gathered}
-\left(p y^{\prime}\right)^{\prime}+q y=\lambda y+y \cdot h(y) \quad \text { on }(a, b), \\
y(a)=0=y(b),
\end{gathered}
$$

admits the following bifurcation theoretic description. Namely, there is a sequence

$$
\lambda_{1}<\lambda_{2}<\lambda_{3}<\cdots<\lambda_{n} \rightarrow+\infty
$$

of simple eigenvalues to the problem

$$
\begin{aligned}
-\left(p z^{\prime}\right)^{\prime}+q z & =\lambda z \quad \text { on }(a, b), \\
z(a) & =0=z(b),
\end{aligned}
$$

such that for each $n$, there is an unbounded continuum $\mathscr{C}_{n}$ in $\mathbf{R} \times\{y \in$ $\left.C^{1}[a, b]: y(a)=0=y(b)\right\}$ of solutions to (2.1)-(2.2) such that $\left(\lambda_{n}, 0\right) \in \mathscr{C}_{n}$. Moreover, the continuum $\mathscr{C}_{n}$ may be written as $\mathscr{C}_{n}=\mathscr{C}_{n}^{+} \cup \mathscr{C}_{n}^{-}$, where $\mathscr{C}_{n}^{+}, \mathscr{C}_{n}^{-}$ are unbounded subcontinua of $\mathscr{C}_{n}$ with the property that $\mathscr{C}_{n}^{+} \cap \mathscr{C}_{n}^{-}=\left\{\left(\lambda_{n}, 0\right)\right\}$. Furthermore, if $(\lambda, y) \in \mathscr{C}_{n}^{+}\left(\mathscr{C}_{n}^{-}\right)$and $(\lambda, y) \neq\left(\lambda_{n}, 0\right), y \not \equiv 0, y$ has exactly $n-1$ simple zeros in $(a, b)$ with $y^{\prime}(a)>0\left(y^{\prime}(a)<0\right)$ and $(-1)^{n} y^{\prime}(b)>$ $0\left((-1)^{n} y^{\prime}(b)<0\right)$. This last fact is a consequence of the uniqueness of initial value problems for $(2.1)$ and the openness in $\left\{y \in C^{1}[a, b]: y(a)=0=y(b)\right\}$ 
of the sets $S_{n}^{+}, S_{n}^{-}$, where $S_{n}^{+}=\left\{y \in C^{1}[a, b]: y(a)=0=y(b), y\right.$ has exactly $n-1$ simple zeros in $(a, b), y^{\prime}(a)>0$, and $\left.(-1)^{n} y^{\prime}(b)>0\right\}$ and $S_{n}^{-}=\left\{y \in C^{1}[a, b]: y(a)=0=y(b), y\right.$ has exactly $n-1$ simple zeros in $(a, b), y^{\prime}(a)<0$, and $\left.(-1)^{n} y^{\prime}(b)<0\right\}$.

We wish to apply Theorem 2.1 of [3] to the situation of (1.2). Using the preceding exposition, in the context of (1.2) the result may be stated as follows:

Theorem 2.1. Suppose $f, g: \mathbf{R}^{2} \rightarrow \mathbf{R}$ are twice continuously differentiable, $f(0,0)=0=g(0,0)$, and that $f(E)$ and $g(E)$ are bounded sets in $\mathbf{R}$ for any bounded set $E \subseteq \mathbf{R}^{2}$. Let $\left(\lambda_{0}, u_{0}\right)$ be a solution to (1.3) such that $\lambda_{0} \neq 0$, $\left(\lambda_{0}, u_{0}\right) \neq\left(\lambda_{n}, 0\right),\left(\lambda_{0}, u_{0}\right) \in \mathscr{C}_{n}$ and such that the boundary value problem

$$
\begin{aligned}
-\left(p_{1} z^{\prime}\right)^{\prime}+q_{1} z & =\lambda_{0} z+f\left(u_{0}, 0\right) z+u_{0} \cdot \frac{\partial f}{\partial u}\left(u_{0}, 0\right) z \quad \text { in }(a, b), \\
z(a) & =0=z(b),
\end{aligned}
$$

has only the solution $z \equiv 0$. Then there is an open interval $V$ about $\lambda_{0}$ and a unique continuous map $u: V \rightarrow\left\{y \in C^{1}[a, b]: y(a)=0=y(b)\right\}$ such that $u\left(\lambda_{0}\right)=u_{0}, u(\lambda) \not \equiv 0$, and $(\lambda, u(\lambda)) \in \mathscr{C}_{n}$ (relative to (1.3)). Let $W=\{(\lambda, u(\lambda)): \lambda \in V\}$. Suppose in addition that $\mu_{0} \in \mathbf{R}$ is such that

$$
\begin{aligned}
-\left(p_{2} w^{\prime}\right)^{\prime}+\left[q_{2}-g\left(u_{0}, 0\right)\right] w & =\mu_{0} w \quad \text { in }(a, b), \\
w(a) & =0=w(b),
\end{aligned}
$$

has a solution $w \not \equiv 0$ with $m-1$ simple zeros in $(a, b)$. Then there is a continuum $\mathscr{C}$ in $\mathbf{R}^{2} \times\left(\left\{y \in C^{1}[a, b]: y(a)=0=y(b)\right\}\right)^{2}$ of solutions to (1.2) which has dimension greater than or equal to 2 (see [1]) at every point. Moreover, $\mathscr{C} \cap(W \times \mathbf{R} \times\{0\}) \neq \varnothing$, and if $W \times \mathbf{R} \times\{0\}$ is viewed as the known or "trivial" sheet of solutions to (1.2), $\mathscr{C}$ is global with respect to this sheet in the Cech cohomological sense of [1]. Furthermore, there is a neighborhood $V_{0}$ of $\lambda_{0}$ in $V$ such that if $\lambda \in V_{0}$ is fixed, there is a $\mu(\lambda) \in \mathbf{R}$ such that the corresponding restriction $\mathscr{C}_{\lambda}$ of $\mathscr{C}$ meets $W \times \mathbf{R} \times\{0\}$ at $(\lambda, \mu(\lambda), u(\lambda), 0)$ and satisfies the global bifurcation alternatives of Rabinowitz with respect to $(\lambda, u(\lambda)) \times \mathbf{R} \times\{0\}$. In particular, $\left(\lambda_{0}, \mu_{0}, u_{0}, 0\right)$ is such a point, and $\mu(\lambda) \rightarrow \mu_{0}$ as $\lambda \rightarrow \lambda_{0}$.

Several observations are in order at this point. First of all, if $(\lambda, \mu, u, v) \in$ $\mathscr{C},(\lambda, \mu, u, v) \notin W \times \mathbf{R} \times\{0\}$ and $(\lambda, \mu, u, v)$ is sufficiently close in $\mathbf{R}^{2} \times$ $\left(\left\{y \in C^{1}[a, b]: y(a)=0=y(b)\right\}\right)^{2}$ to $\left(\lambda_{0}, \mu_{0}, u_{0}, 0\right)$ then $u$ has $n-1$ simple zeros in $(a, b)$ and $v$ has $m-1$ simple zeros in $(a, b)$. More specifically, $u \in S_{n}^{+}\left(S_{n}^{-}\right)$if $u_{0} \in S_{n}^{+}\left(S_{n}^{-}\right)$. However, $v$ may be in either $S_{m}^{+}$or $S_{m}^{-}$. In fact, for $\lambda$ fixed sufficiently near $\lambda_{0}$ and $\varepsilon>0$ sufficiently small, $\mathscr{C}_{\lambda} \cap B((\lambda, \mu(\lambda), u(\lambda), 0) ; \varepsilon)$ may be expressed as $\mathscr{C}_{\lambda}^{+} \cup \mathscr{C}_{\lambda}^{-}$, where $\mathscr{C}_{\lambda}^{+}, \mathscr{C}_{\lambda}^{-}$ are continua such that $\mathscr{C}_{\lambda}^{-} \cap \mathscr{C}_{\lambda}^{-}=\{(\lambda, \mu(\lambda), u(\lambda), 0)\}$ and if $(\lambda, \mu, u, v) \in$ $\mathscr{C}_{\lambda}^{+}\left(\mathscr{C}_{\lambda}^{-}\right), v \in S_{m}^{+}\left(S_{m}^{-}\right)$. (Here $B((\lambda, \mu(\lambda), u(\lambda), 0) ; \varepsilon)$ is the ball of radius $\varepsilon$ about $(\lambda, \mu(\lambda), u(\lambda), 0)$ in $\{\lambda\} \times \mathbf{R} \times\left(\left\{y \in C^{1}[a, b]: y(a)=0=y(b)\right\}\right)^{2}$.) That such is the case follows readily from the fact that $\mu_{0}$ is simple and that $f$ 
and $g$ are smooth enough to apply the local bifurcation theory of Crandall and Rabinowitz [8] to this situation. (See also, for example, [5, Section 3] and [1, Theorem 3.12]). Secondly, the structure of the equations in system (1.2), uniqueness of initial value problems, and the openness in $\left\{y \in C^{1}[a, b]: y(a)=\right.$ $0=y(b)\}$ of $S_{n}^{ \pm}$and $S_{m}^{ \pm}$guarantee that the nodal structure of $(u, v)$ is maintained as one moves along $\mathscr{C}$ until $u$ or $v$ becomes 0 . Thirdly, since for any $u_{0}$

$$
\begin{aligned}
-\left(p_{2} w^{\prime}\right)^{\prime}+\left[q_{2}-g\left(u_{0}, 0\right)\right] w & =\mu w \quad \text { in }(a, b), \\
w(a) & =0=w(b),
\end{aligned}
$$

has an increasing sequence of simple eigenvalues $\mu_{1}<\mu_{2}<\cdots$ and a corresponding sequence of eigenfunctions $w_{1}, w_{2}, \ldots$, where $w_{j}$ has $j-1$ simple zeros in $(a, b)$, condition (2.6) can always be met. Consequently, Theorem 2.1 may be invoked so long as the existence of a $\left(\lambda_{0}, u_{0}\right)$ satisfying $(2.5)$ can be established. The heart of this section is to find conditions on $f$ to guarantee such. Finally, we should note that there is an analogous formulation of Theorem 2.1 requiring that

$$
\begin{aligned}
-\left(p_{2} z^{\prime}\right)^{\prime}+q_{2} z & =\mu_{0} z+g\left(0, v_{0}\right) z+v_{0} \frac{\partial g}{\partial v}\left(0, v_{0}\right) z, \quad \text { in }(a, b), \\
z(a) & =0=z(b),
\end{aligned}
$$

have only the trivial solution.

We now turn to the task of determining conditions on $f$ which guarantee that (1.3) has solutions $\left(\lambda_{0}, u_{0}\right)$ for which $(2.5)$ has only the trivial solution. First of all, observe that the Crandall-Rabinowitz local bifurcation theory [8] implies that $\mathscr{C}_{n}$ (with respect to (1.3)) in a neighborhood of $\left(\lambda_{n}, 0\right)$ (in $\mathbf{R} \times\{y \in$ $\left.\left.C^{1}[a, b]: y(a)=0=y(b)\right\}\right)$ may be expressed as

$$
\{(\lambda(t), u(t)): t \in(-\delta, \delta)\}
$$

where $(\lambda(0), u(0))=\left(\lambda_{n}, 0\right)$. Moreover, $\lambda(t), u(t)$ are smooth functions of $t$ with derivatives $\lambda_{t}(t), u_{t}(t)$ satisfying

$$
\begin{aligned}
-\left(p_{1} u_{t}^{\prime}\right)^{\prime}+q_{1} u_{t} & =\lambda_{t} u+\lambda u_{t}+f(u, 0) u_{t}+u \frac{\partial f}{\partial u}(u, 0) u_{t}, \\
u_{t}(a) & =0=u_{t}(b) .
\end{aligned}
$$

Suppose now for some $(\lambda(t), u(t))$ there is a $w \not \equiv 0$ satisfying (2.5). An integration by parts argument shows us that

$$
\lambda_{t}(t) \int_{a}^{b} u(t)(x) w(x) d x=0
$$


We may assume that $\|w\|=1$, where \|\| denotes the usual norm in $C^{1}[a, b]$. Let us assume that we have $t_{n} \rightarrow 0, t_{n} \neq 0$ and $w_{n}$ with $\left\|w_{n}\right\|=1$ satisfying

$$
\begin{aligned}
-\left(p_{1} w_{n}^{\prime}\right)^{\prime}+q_{1} w_{n}= & \lambda\left(t_{n}\right) w_{n}+f\left(u\left(t_{n}\right), 0\right) w_{n} \\
& +u\left(t_{n}\right) \cdot \frac{\partial f}{\partial u}\left(u\left(t_{n}\right), 0\right) w_{n} \quad \text { in }(a, b), \\
w_{n}(a)= & 0=w_{n}(b) .
\end{aligned}
$$

Since $u\left(t_{n}\right) \rightarrow 0$ and $\lambda\left(t_{n}\right) \rightarrow \lambda_{n}$ as $n \rightarrow \infty$, it follows from (2.8) and a compactness argument that a subsequence $w_{n_{i}}$ of $w_{n}$ converges to $w$, where $\|w\|=1$ and $w$ solves

$$
\begin{aligned}
-\left(p_{1} w^{\prime}\right)^{\prime}+q_{1} w & =\lambda_{n} w \quad \text { in }(a, b) \\
w(a) & =0=w(b) .
\end{aligned}
$$

A similar argument shows that a subsequence $u\left(t_{n_{i_{j}}}\right) /\left\|u\left(t_{n_{i_{j}}}\right)\right\|$ of $u\left(t_{n_{i}}\right) /\left\|u\left(t_{n_{i}}\right)\right\|$ converges to $w$ or $-w$. Consequently,

$$
\lim _{j \rightarrow \infty} \int_{a}^{b}\left(u\left(t_{n_{i_{j}}}\right) /\left\|u\left(t_{n_{i_{j}}}\right)\right\|(x)\right) w_{n_{i_{j}}}(x) d x= \pm \int_{a}^{b} w^{2}(x) d x \neq 0 .
$$

So $\int_{a}^{b} u\left(t_{n_{i_{j}}}\right)(x) w_{n_{i_{j}}}(x) d x \neq 0$ if $j$ is sufficiently large, since $\left\|u\left(t_{n_{i_{j}}}\right)\right\|$ is a nonzero constant. Now we see from (2.7) that we can have a nontrivial solution $w_{n_{i j}}$ of (2.8) only if

$$
\lambda_{t}\left(t_{n_{i_{j}}}\right)=0
$$

for all $j$ sufficiently large. As a consequence, so long as there is a sequence $t_{n} \rightarrow$ 0 with $\lambda_{t}\left(t_{n}\right) \neq 0$ for all $n$, there are infinitely many solutions $\left(\lambda\left(t_{n}\right), u\left(t_{n}\right)\right)$ of (1.3) for which (2.5) has only the trivial solution. Any such solution may play the role of $\left(\lambda_{0}, u_{0}\right)$ in Theorem 2.1. We have established the following result on the range of applicability of Theorem 2.1.

Theorem 2.2. Consider (1.3). Suppose that $f$ is such that there is not a neighborhood $B\left(\left(\lambda_{n}, 0\right) ; \varepsilon\right)$ in $\mathbf{R} \times\left\{u \in C^{1}[a, b]: u(a)=0=u(b)\right\}$ with the property that the projection of $\left.\mathscr{C}_{n} \cap B\left(\left(\lambda_{n}, 0\right) ; \varepsilon\right)\right)$ into $\mathbf{R}$ is $\left\{\lambda_{n}\right\}$. Then there is a $\left(\lambda_{0}, u_{0}\right) \in \mathscr{C}_{n}$ so that (2.5) has only the trivial solution. Moreover, if $\mu_{1}<\mu_{2}<\cdots$ denotes the sequence of eigenvalues to (2.6), there is a continuum $\mathscr{C}^{m}$ of solutions to (1.2) as described in Theorem 2.1 emanating from $\left(\lambda_{0}, \mu_{m}, u_{0}, 0\right)$.

We should make some comments at this point. Once again, there is a companion result to Theorem 2.2 which is expressed in terms of the solution set to (1.4) in place of (1.3). In addition, it should be noted that the restriction on $f$ (or $g$ ) can be described in terms of bifurcation diagrams. Namely, the requirement is that $\mathscr{C}_{n}$ relative to (1.3) (or (1.4)) does not emanate "vertically" from $\left(\lambda_{n}, 0\right)$ (or $\left.\left(\mu_{n}, 0\right)\right)$. Consequently, Theorem 2.2 allows us to treat a great many interesting examples in (1.2). For instance, if $f(u, 0)=\alpha u$ or $\alpha u^{2}$, where $\alpha \neq 0$, Theorem 2.2 applies for any choice of $n \in \mathbf{Z}^{+}$. Hence, we get 
continua of solutions $(u, v)$ to (1.2) where $u$ has $n-1$ simple zeros in $(a, b)$ and $v$ has $m-1$ simple zeros in $(a, b)$ for any choice of $(n, m) \in\left(\mathbf{Z}^{+}\right)^{2}$. However, Theorem 2.2 does not apply to a situation where both $f(u, 0)$ and $g(0, v)$ are identically zero. For example, our analysis does not treat

$$
\begin{array}{ll}
-u^{\prime \prime}=\lambda u-u v & \text { in }(0,1), \\
-v^{\prime \prime}=\mu v+u v & \text { in }(0,1), \\
u(0)=0=u(1), & \\
v(0)=0=v(1) . &
\end{array}
$$

\section{LINKING PHENOMENA}

If both $\mathscr{C}_{n}$ relative to (1.3) and $\mathscr{C}_{m}$ relative to (1.4) satisfy the hypotheses of Theorem 2.2, there is a continuum of nontrivial solutions $(u, v)$ to $(1.2)$ with $u$ having $n-1$ simple zeros and $v$ having $m-1$ simple zeros arising from solutions to (1.2) having the form $(u, 0)$, and, in addition, one arising from solutions to $(1.2)$ having the form $(0, v)$. It is natural to ask if these continua are linked together. Certainly, some linking in a neighborhood of $\left(\lambda_{n}, \mu_{m}, 0,0\right)$ could be expected. However, the question we wish to address in this section is one that can give global as well as local information about the continua. Namely, suppose $\left(\lambda_{0}, u_{0}\right) \in \mathscr{C}_{n}$ with $u_{0} \not \equiv 0$ so that $(2.5)$ has only the trivial solution and that $\mu_{m}$ is the $m$ th eigenvalue of (2.6). Suppose also that we restrict $\lambda=\lambda_{0}$ and follow the continuum of solutions to (1.2) $\mathscr{C}_{\lambda_{0}}$ emanating from $\left(\lambda_{0}, \mu_{m}, u_{0}, 0\right)$ in $\left\{\lambda_{0}\right\} \times \mathbf{R} \times\left(\left\{y \in C^{1}[a, b]: y(a)=0=y(b)\right\}\right)^{2}$. We know from Section 2 that the solutions to (1.2) which emanate from $\left(\lambda_{0}, \mu_{m}, u_{0}, 0\right)$ have $n-1$ simple zeros in $(a, b)$ for $u$ and $m-1$ simple zeros in $(a, b)$ for $v$. Moreover, we know that this nodal structure is maintained until $u$ or $v$ becomes zero. The question we wish to address is: does the continuum $\mathscr{C}_{\lambda_{0}}$ contain a solution to (1.2) of the form $\left(\lambda_{0}, \mu, 0, v\right)$ with $v \not \equiv 0$ ?

We know from Theorem 2.2 that either $\mathscr{C}_{\lambda_{0}}$ is unbounded or there is $\left(\lambda_{0}, \bar{\mu}\right.$, $\left.u_{0}, 0\right) \in \mathscr{C}_{\lambda_{0}}$ with $\bar{\mu} \neq \mu_{m}$. (It is important to note that $\left(\lambda_{0}, \mu, 0, v\right)$ and $\left(\lambda_{0}, \mu, u^{*}, 0\right), u^{*} \neq u_{0}$, are not "trivial solutions" in this setting. See the proof of [3, Theorem 2.1].) Moreover, we know from preservation of nodal structure that $\left(\lambda_{0}, \bar{\mu}, u_{0}, 0\right)$ may not be a limit point in $\mathscr{C}_{i_{0}}$ of solutions to (1.2) of $(n, m)$ nodal type. However, to answer the question in the affirmative we still need more information on $\mathscr{C}_{\lambda_{0}}$. The two principal additional pieces of information that we need are that ${\stackrel{\mathscr{C}}{\lambda_{0}}}_{\hat{x}}$ cannot become unbounded unless $\mu$ becomes unbounded and that if $\mu$ does become unbounded, not both $u$ and $v$ can be nonzero.

We must now determine conditions on $f$ and $g$ which enable us to provide this additional information on $\mathscr{C}_{\lambda_{0}}$. We begin with the following lemma.

Lemma 3.1. Let $q_{1}, q_{2} \geq 0$. Suppose that there are continuous functions $h$, $k: \mathbf{R} \rightarrow[0, \infty)$ so that 


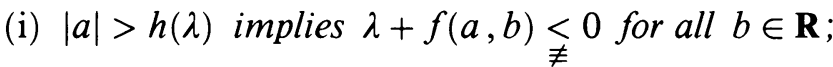

(ii) $|b|>k(\mu)$ implies $\mu+g(a, b) \underset{\not \equiv}{\gtrless}$ for all $a \in \mathbf{R}$.

Then if $(\lambda, \mu, u, v)$ is a solution to (1.2), $\|u\|_{\infty} \leq h(\lambda)$ and $\|v\|_{\infty} \leq k(\mu)$.

Proof. The result follows from the maximum principle [7]. See also [6].

If $f$ and $g$ satisfy (i) and (ii) of Lemma 3.1, then there is a continuous function $M: \mathbf{R}^{2} \rightarrow[0, \infty)$ so that if $(\lambda, \mu, u, v)$ is any solution of $(1.2)$, then

$$
\|u\|_{\infty}+\left\|u^{\prime}\right\|_{\infty}+\|v\|_{\infty}+\left\|v^{\prime}\right\|_{\infty} \leq M(\lambda, \mu) \text {. }
$$

Consequently, $\mathscr{C}_{\lambda_{0}}$ can become unbounded only as $\mu$ becomes unbounded.

We now need to know that if $\mu$ becomes unbounded and $\left(\lambda_{0}, \mu, u, v\right) \in \mathscr{C}_{\lambda_{0}}$, then either $u=0$ of $v=0$. To this end, we make the additional assumptions on (1.2) that

$$
p_{1} \geq p_{2} ; \quad q_{1} \geq q_{2}
$$

and

$$
\left.\begin{array}{l}
f(a, b) \leq 0 \\
g(a, b) \leq 0
\end{array}\right\} \quad \text { for all }(a, b) \in \mathbf{R}^{2} .
$$

We have the following lemma.

Lemma 3.2. Let the conditions of Lemma 3.1 obtain. Suppose that (3.1) and (3.2) hold. For $i=1,2$, let $\lambda_{1}^{i}, \lambda_{2}^{i}, \ldots$, denote the eigenvalues of

$$
\begin{aligned}
-\left(p_{i} w^{\prime}\right)^{\prime}+q_{i} w & =\lambda w \quad \text { in }(a, b), \\
w(a) & =0=w(b) .
\end{aligned}
$$

Suppose there is a continuous function $\varphi:\left[\lambda_{1}^{1}, \infty\right) \rightarrow[0, \infty)$ such that $\mu>\varphi(\lambda)$ implies

$$
\lambda+f(a, b)<\mu+g(a, b)
$$

for all $(a, b) \in \mathbf{R}^{2}$ with $|a| \leq h(\hat{\lambda})$ and $|b| \leq k(\mu)$. Then if $(\lambda, \mu, u, v)$ is a solution of (1.2) where $u$ has $n-1$ simple zeros in $(a, b), \mu>\varphi(\lambda)$, and $v \not \equiv 0, v$ has at least $n$ simple zeros in $(a, b)$.

Proof. Let $(\lambda, \mu, u, v)$ be a solution to (1.2) as above. Since $u \neq \equiv 0, v \not \equiv$ 0 , (3.2) and the Sturm Comparison Theorem imply $\lambda \geq \lambda_{1}^{1}$ and $\mu \geq \lambda_{1}^{2}$. Conditions (3.1), (3.3), and Lemma 3.1 imply that

is a strict Sturm majorant of

$$
p=p_{2}, \quad q=\mu+g(u, v)-q_{2}
$$

$$
\tilde{p}=p_{1}, \quad \tilde{q}=\lambda+f(u, v)-q_{1} .
$$

The result now follows from the Sturm Comparison Theorem.

Corollary 3.3. If (3.1), (3.2), and (3.3) hold and $(\lambda, \mu, u, v)$ is a solution of (1.2), where $u$ has $n-1$ simple zeros and $v$ has $m-1$ simple zeros with $m \leq n$, then $\mu \in\left[\lambda_{1}^{2}, \varphi(\lambda)\right]$.

We may now prove the following result. 
Theorem 3.4. Suppose that the conditions of Lemma 3.1 and Lemma 3.2 are met. Suppose that $\left(\lambda_{0}, u_{0}\right) \in \mathscr{C}_{n}$ with $u_{0} \not \equiv 0$ and $\lambda_{0} \neq \lambda_{n}$ so that (2.5) has only the trivial solution and that $\mu_{m}$ is the $m$ th eigenvalue of (2.6), where $m \leq n$. Let $\mathscr{C}_{\lambda_{0}}$ denote the continuum of solutions to (1.2) emanating from $\left(\lambda_{0}, \mu_{m}, u_{0}, 0\right)$ in $\left\{\lambda_{0}\right\} \times \mathbf{R} \times\left(\left\{y \in C^{1}[a, b]: y(a)=0=y(b)\right\}\right)^{2}$, whose existence is guaranteed by Theorem 2.2. Suppose also that if $u$ is any solution to (1.3) with $\lambda=\lambda_{0}$ and having $n-1$ simple zeros in $(a, b)$, then $u=u_{0}$ or $u^{\prime}(a) \cdot u_{0}^{\prime}(a)<0$. Then there is $a \bar{v}$ with $m-1$ simple zeros in $(a, b)$ and $\bar{\mu} \in\left[\lambda_{m}^{2}, \varphi\left(\lambda_{0}\right)\right]$ so that $\left(\lambda_{0}, \bar{\mu}, 0, \bar{v}\right) \subset \mathscr{C}_{\lambda_{0}}$.

Proof. Let $N=\left\{(u, v) \in\left(\left\{C^{1}[a, b]: y(a)=0=y(b)\right\}\right)^{2}: u\right.$ has $n-1$ simple zeros in $(a, b), u^{\prime}(a) \cdot u_{0}^{\prime}(a)>0, u^{\prime}(b) \cdot u_{0}^{\prime}(b)>0, v$ has $m-1$ simple zeros in $(a, b)$, and $\left.v^{\prime}(a) \cdot v^{\prime}(b) \neq 0\right\}$ and let $\mathscr{N}=\left\{\lambda_{0}\right\} \times \mathbf{R} \times N$. For $\varepsilon>0$, let $T_{\varepsilon}=\left\{\left(\lambda_{0}, \mu, u, v\right) \in \mathscr{C}_{\lambda_{0}}:\left\|\left(\lambda_{0}, \mu, u, v\right)-\left(\lambda_{0}, \mu_{m}, u_{0}, 0\right)\right\|_{(\leftarrow)[a, b])^{2} \times \mathbf{R}}=\varepsilon\right\}$. It follows from Theorem 2.2 that $T_{\varepsilon} \subseteq \mathcal{N}$ for all $\varepsilon>0$ and sufficiently small.

We first show that there is an $\varepsilon_{0}>0$ so that $T_{\varepsilon_{0}} \nsubseteq \mathscr{N}$. Suppose to the contrary that $T_{\varepsilon} \subseteq \mathscr{N}$ for all $\varepsilon>0$. Then Theorem 2.2 implies that $T_{\varepsilon} \neq \varnothing$ for all $\varepsilon>0$. (If not, there is $\left(\lambda_{0}, \mu^{*}, u_{0}, 0\right) \in \mathscr{C}_{\lambda_{0}}$ with $\mu^{*} \neq \mu_{m}$, and consequently, $\left(\lambda_{0}, \mu, u, v\right) \in \mathscr{C}_{\lambda_{0}}$ with $v$ having $k-1$ simple zeros in $(a, b)$, $k \neq m$, a contradiction.) Corollary 3.3 and the proof of Lemma 3.2 guarantee that if $\left(\lambda_{0}, \mu, u, v\right) \in \mathscr{C}_{\lambda_{0}} \cap \mathscr{N}$, then $\mu \in\left[\lambda_{m}^{2}, \varphi\left(\lambda_{0}\right)\right]$. It now follows from Lemma 3.1 that there is a $\mathrm{C}>0$ so that $\left(\lambda_{0}, \mu, u, v\right) \in \mathscr{C}_{\lambda_{0}} \cap \mathcal{N}$ implies $\left\|\left(\lambda_{0}, \mu, u, v\right)-\left(\lambda_{0}, \mu_{m}, u_{0}, 0\right)\right\|_{\left(F^{\prime}[a, b]\right)^{2} \times \mathbf{R}} \leq \mathrm{C}$. But then $T_{\varepsilon}=\varnothing$ if $\varepsilon>\mathrm{C}$, a contradiction.

So there is an $\varepsilon_{0}>0$ with $T_{\varepsilon_{0}} \backslash \mathscr{N} \neq \varnothing$. Hence there is $\left(\lambda_{0}, \bar{\mu}, \bar{u}, \bar{v}\right) \in$ $\mathscr{C}_{\lambda_{0}}$ with the property that there is a sequence $\left\{\left(\lambda_{0}, \mu_{k}, u_{k}, v_{k}\right)\right\}_{k=1}^{\infty} \in \mathscr{C}_{\lambda_{0}} \cap \mathcal{N}$ converging to $\left(\lambda_{0}, \bar{\mu}, \bar{u}, \bar{v}\right)$ while $(\bar{u}, \bar{v}) \in \partial N$ and $\|\left(\lambda_{0}, \bar{\mu}, \bar{u}, \bar{v}\right)-\left(\lambda_{0}, \mu_{m}\right.$, $\left.u_{0}, 0\right) \|_{\left(\kappa^{\prime}[a, b]\right)^{2} \times \mathbf{R}} \geq \delta>0$. Hence either $\bar{u} \equiv 0$ or $\bar{v} \equiv 0$. Suppose $\bar{v} \equiv$ 0 . Then $\bar{u} \not \equiv 0$ since $\lambda_{0} \neq \lambda_{n}$. So $\bar{u}$ has $n-1$ simple zeros in $(a, b)$, $\vec{u}^{\prime}(a) \cdot u_{0}^{\prime}(a)>0$, and $\bar{u}^{\prime}(b) \cdot u_{0}^{\prime}(b)>0$, since $\left(\lambda_{0}, \bar{\mu}, \bar{u}, \bar{v}\right) \in \mathscr{C}_{\lambda_{0}}$. Hence $\bar{u} \equiv u_{0}$. But now $\bar{\mu} \neq \mu_{m}$, since $\left\|\left(\lambda_{0}, \bar{\mu}, \bar{u}, \bar{v}\right)-\left(\lambda_{0}, \mu_{m}, u_{0}, 0\right)\right\|_{\left(K^{\prime}[a, b]\right)^{2} \times \mathbf{R}} \geq \delta>0$. Since $v_{k}$ has $m-1$ simple zeros in $(a, b)$ for all $k \in \mathbf{Z}^{+}$, this last is not possible. So $\bar{v} \not \equiv 0$ and $\bar{u} \equiv 0$. By the Sturm Comparison Theorem, $\bar{\mu} \geq \lambda_{m}^{2}$. If $\bar{\mu}>\varphi\left(\lambda_{0}\right)$, there is $\left(\lambda_{0}, \mu_{k}, u_{k}, v_{k}\right) \in \mathscr{C}_{\lambda_{0}}$ with $u_{k}$ having $n-1$ simple zeros in $(a, b)$ and $v_{k}$ having $m-1$ simple zeros in $(a, b)$ while $\mu_{k}>\varphi\left(\lambda_{0}\right)$, a contradiction to Corollary 3.3. So $\bar{\mu} \in\left[\lambda_{m}^{2}, \varphi\left(\lambda_{0}\right)\right]$.

There is of course an analogous result to Theorem 3.4 wherein the roles of the first and second equations in (1.2) are reversed provided the inequalities in (3.1) are also reversed. Moreover, we should note that condition (3.2) can be weakened to

$$
\left.\begin{array}{l}
f(a, b) \leq K \\
g(a, b) \leq K
\end{array}\right\} \quad \text { for all }(a, b) \in \mathbf{R}^{2}
$$

where $K>0$ provided that in Theorem $3.4, \bar{\mu} \in\left[\lambda_{m}^{2}-K, \varphi\left(\lambda_{0}\right)\right]$. 


\section{AN EXAMPLE}

Consider the system

$$
\begin{aligned}
& -u^{\prime \prime}=\lambda u-\left(\alpha u^{2}+\beta v^{2}\right) u \\
& -v^{\prime \prime}=\mu v-\left(\gamma u^{2}+\delta v^{2}\right) v \\
& u(a)=u(b)=0=v(a)=v(b),
\end{aligned}
$$

where $\alpha, \beta, \gamma, \delta$ are positive constants. It is evident that (4.1) is a special case of (1.2) with $f(u, v)=-\left(\alpha u^{2}+\beta v^{2}\right)$ and $g(u, v)=-\left(\gamma u^{2}+\delta v^{2}\right)$. Note that (1.3) and (1.4) in this setting are

$$
\begin{aligned}
& -u^{\prime \prime}=\lambda u-\alpha u^{3} \quad \text { in }(a, b), \\
& u(a)=0=u(b),
\end{aligned}
$$

and

$$
\begin{aligned}
& -v^{\prime \prime}=\mu v-\delta v^{3} \quad \text { in }(a, b), \\
& v(a)=0=v(b),
\end{aligned}
$$

respectively. It follows from the Sturm Comparison Theorem that solutions to (4.2) (or (4.3)) with $n-1$ simple zeros satisfy $\lambda>\sigma_{n}$ (or $\mu>\sigma_{n}$ ), where $\sigma_{1}<\sigma_{2}<\sigma_{3} \cdots$ are the eigenvalues of

$$
\begin{aligned}
& -w^{\prime \prime}=\sigma w \quad \text { in }(a, b), \\
& w(a)=0=w(b) .
\end{aligned}
$$

Consequently, $f$ and $g$ satisfy the hypotheses of Theorem 2.2. Thus there are continua of solutions $(\lambda, \mu, u, v)$ to (4.1) with $u$ having $n-1$ simple zeros in $(a, b)$ and $v$ having $m-1$ simple zeros in $(a, b)$ which emanate from solutions to (4.1) of the form $(\lambda, \mu, u, 0)$, where $u$ has $n-1$ simple zeros in $(a, b)$ and $(\lambda, u)$ satisfies (4.2) and from solutions to (4.1) of the form $(\lambda, \mu, 0, v)$, where $v$ has $m-1$ simple zeros in $(a, b)$ and $(\mu, v)$ satisfies (4.3).

We aim to show that linking as in Section 3 occurs for (4.1). Observe that Lemma 3.1 holds with $h$ and $k$ given by

$$
h(\lambda)=\left\{\begin{aligned}
0, & \lambda \leq 0, \\
\sqrt{\frac{\lambda}{\alpha}}, & \lambda>0,
\end{aligned}\right.
$$

and

$$
k(\mu)=\left\{\begin{aligned}
0, & \mu \leq 0, \\
\sqrt{\frac{\mu}{\delta}}, & \mu>0 .
\end{aligned}\right.
$$

It is also evident that equality obtains in (3.1) and that (3.2) holds. 
Now let $\lambda \geq \sigma_{1}, \mu \geq \sigma_{1},|a| \leq \sqrt{\lambda / \alpha}$, and $|b| \leq \sqrt{\mu / \delta}$. Suppose that in addition $\beta<2 \delta$. Then $|\beta-\delta| / \delta<1$. So

$$
\mu>\frac{2+\frac{\nu}{\alpha}}{1-\frac{|\beta-\delta|}{\delta}} \lambda \Rightarrow\left(1-\frac{|\beta-\delta|}{\delta}\right) \mu>\left(2+\frac{\gamma}{\alpha}\right) \lambda \Rightarrow \mu-\frac{\gamma}{\alpha} \lambda>2 \lambda+\frac{|\beta-\delta|}{\delta} \mu .
$$

But now

$$
\begin{aligned}
\lambda-\alpha a^{2}-(\beta-\delta) b^{2} & \leq \lambda+\alpha a^{2}+|\beta-\delta| b^{2} \\
& \leq 2 \lambda+\frac{|\beta-\delta|}{\delta} \mu
\end{aligned}
$$

and $\mu-\gamma a^{2} \geq \mu-\frac{2}{\alpha} \lambda$. By (4.5), $\mu>\frac{2+\frac{z}{\alpha}}{1-\frac{|\beta-\delta|}{\delta}} \lambda$ implies $\mu-\gamma a^{2}>\lambda-\alpha a^{2}-$ $(\beta-\delta) b^{2}$, which is equivalent to

$$
\mu+g(a, b)>\lambda+f(a, b)
$$

for $|a| \leq \sqrt{\lambda / \alpha},|b| \leq \sqrt{\mu / \delta}$. Hence if $\beta<2 \delta$, (3.3) is met by

$$
\varphi(\lambda)=\frac{2+\frac{\gamma}{\alpha}}{1-\frac{|\beta-\delta|}{\delta}} \lambda
$$

If $\gamma<2 \alpha$, we get a companion result for $\mu$ with

$$
\varphi(\mu)=\frac{2+\frac{\delta}{\beta}}{1-\frac{|\alpha-\gamma|}{\alpha}} \mu .
$$

As a consequence, Theorem 3.4 implies that the continua described above link solutions to (4.1) of the form $(\lambda, \mu, u, 0)$ where $u$ has $n-1$ simple zeros in $(a, b)$ to solutions of $(4.1)$ of the form $(\lambda, \mu, 0, v)$ where $v$ has $m-1$ simple zeros in $(a, b)$ for any choice of $(n, m) \in\left(\mathbf{Z}^{+}\right)^{2}$ provided the uniqueness hypothesis of Theorem 3.4 is met for (4.2) and (4.3). It suffices to consider (4.2).

Since there are no solutions to (4.2) having $n-1$ simple zeros in $(a, b)$ if $\lambda=\sigma_{n}$ and since $\|u\|_{\infty} \leq \sqrt{\frac{\lambda}{\alpha}}$ for any solution, the only solutions to (4.2) with $n-1$ simple zeros in $(a, b)$ for $\lambda$ sufficiently near $\sigma_{n}$ are those given by the Crandall-Rabinowitz theorem [8]. We know that these solutions can be expressed as $(\lambda(t), u(t))$ with

$$
u(t)=t(w+r(t)) .
$$

Here $w$ satisfies

$$
\begin{gathered}
-w^{\prime \prime}=\sigma_{n} w \text { in }(a, b) \\
w(a)=0=w(b)
\end{gathered}
$$

$w^{\prime}(a)>0$ and $\int_{a}^{b} w^{2} d x=1$, while $r(t)$ lies in a complement of span $\{w\}$ in $\left\{y \in \mathrm{C}^{1}[a, b] ; y(a)=0=y(b)\right\}$ and $r(0)=0$. Moreover, the derivatives of $\lambda$ 
and $u$ with respect to $t$, denoted $\lambda_{t}$ and $u_{t}$, satisfy

$$
\begin{gathered}
-u_{t}^{\prime \prime}=\lambda_{t} u+\lambda u_{t}-3 \alpha u^{2} u_{t} \text { in }(a, b) \\
u_{t}(a)=0=u_{t}(b) .
\end{gathered}
$$

If $\left(\lambda_{k}, u_{k}\right)$ and $\left(\lambda_{k}, u_{k}^{*}\right)$ are distinct solutions to (4.2) having $n-1$ simple zeros in $(a, b)$, satisfying $u_{k}^{\prime}(a) \cdot\left(u_{k}^{*}\right)^{\prime}(a)>0$, and ccnverging to $\left(\sigma_{n}, 0\right)$, there are $t_{k}, t_{k}^{*}$ so that $u_{k}=u\left(t_{k}\right), u_{k}^{*}=u\left(t_{k}^{*}\right)$, and $\lambda\left(t_{k}\right)=\lambda_{k}=\lambda\left(t_{k}^{*}\right)$. It follows from (4.6) and the fact that $u_{k}^{\prime}(a) \cdot\left(u_{k}^{*}\right)^{\prime}(a)>0$ that $t_{k} \cdot t_{k}^{*}>0$ and that $t_{k}$ and $t_{k}^{*}$ converge to 0 . So there is a sequence $\left\{\tilde{t}_{k}\right\}_{k=1}^{\infty}, \tilde{t}_{k} \neq 0$ so that $\lambda_{t}\left(\tilde{t}_{k}\right)=0$. From (4.2) and (4.7) we get via integration by parts that

$$
\lambda_{t}(t) \int_{a}^{b} u^{2} d x=2 \alpha \int_{a}^{b} u^{3} u_{t} d x
$$

By (4.6), $\frac{u(t)}{t}$ and $u_{t}$ converge to $w$ in the topology of $C^{1}[a, b]$. Hence $\lim _{t \rightarrow 0} \int_{a}^{b}\left(\frac{u}{t}\right)^{3} u_{t} d x=\int_{a}^{b} w^{4} d x$. So $\int_{a}^{b} u^{3} u_{t} d x$ is positive for $t>0$ and sufficiently small and negative for $t<0$ and sufficiently small. The same is true for $\lambda_{t}(t)$ by $(4.8)$, a contradiction to $\lambda_{t}\left(\tilde{t}_{k}\right)=0$ for $k=1,2,3, \ldots$. Therefore, the uniqueness assertion of Theorem 3.4 is met and the results of Section 3 apply to (4.1).

\section{REFERENCES}

1. J. C. Alexander and S. S. Antman, Global and local behavior of bifurcating multidimensional continua of solutions for multiparameter nonlinear eigenvalue problems, Arch. Rational Mech. Anal. 76 (1981), 339-354.

2. J. Blat and K. J. Brown, Bifurcation of steady-state solutions in prederator-prey and competition systems, Proc. Roy. Soc. Edinburgh Sect. A 97 (1984), 21-34.

3. R. S. Cantrell, Global higher bifurcations in coupled systems of nonlinear eigenvalue problems, Proc. Roy. Soc. Edinburgh Sect. A 106 (1987), 113-120.

4. __ A switch in nodal structure in coupled systems of nonlinear Sturm-Liouville boundary value problems, Rocky Mountain J. Math., (to appear).

5. R. S. Cantrell and C. Cosner, On the steady-state problem for the Volterra-Lotka competition model with diffusion, Houston J. Math. 13 (1987), 337-352.

6. M. G. Crandall and P. H. Rabinowitz, Nonlinear Sturm-Liouville eigenvalue problems and topological degree, J. Math. Mech. 19 (1970), 1083-1102.

7. M. H. Protter and H. F. Weinberger, Maximum principles in differential equations, PrenticeHall, Englewood Cliffs, N.J., 1967.

8. P. H. Rabinowitz, Some aspects of nonlinear eigenvalue problems, Rocky Mountain J. Math. 3 (1973), 161-202.

Department of Mathematics and Computer Science, The University of Miami, Coral GABLES, Florida 33124 